Special Article

\title{
New frontiers in transfusion medicine: gene therapy
}

Patrick Hervé ${ }^{1,2}$

Pierre Tiberghien ${ }^{2}$

\section{Introduction}

Techniques of separation, purification as well as cryopreservation of marrow, blood, cord blood or tissue cells have been developed in blood banks. Transfusion medicine, over the last 20 years, has advanced radically from total blood transfusion to cell and ex vivo gene therapy, going through different stages, including the production of well-defined cell products (red blood cells, platelets, granulocytes), T-cell depletion, as well as cell purification and expansion (hematopoietic stem cells [HSC], immunocompetent cells). Given the experience acquired by blood transfusion centers, one of the main objectives is to adapt modern transfusion medicine for these new types of blood or marrow or tissue products. Transfusion medicine is facing real challenges in various fields of biotechnology, these novel approaches represent an extraordinary therapeutic potential and development tool $(1,2)$.

The list of diseases - hematological or otherwise - in which gene therapy finds applications has lengthened as a result of the advances achieved in the fields of molecular biology and pathology, converging in the development of improved vectors to bring gene transfer into the clinic. Hematopoietic cells were
Relevant aspects in the advances in transfusion medicine using gene therapy are related in this report. Rev.bras.hematol.hemoter., 2000(22) (3): 362-367

Key words: Transfusion medicine, gene therapy

first chosen, as they are accessible for ex vivo manipulation and can be easily engrafted. In 1990, Blaese et al. transplanted autologous peripheral blood leukocytes modified by retroviral transfer of the adenosine deaminase gene into children with inherited SCID (3).

Extensive basic research has long been carried out in the areas of vector design (retrovirus, adenovirus) (4). Retroviruses represent a vector of choice for sustained transgenesis in actively renewing tissues such as the hematopoietic system. In contrast, the small DNA containing adeno-associated viruses (AAVs) represents an attractive vector system, as they can infect a broad range of target cells.

This paper provides an overview of the variety of medical applications for gene transfer technology. A potential role of transfusion medicine is expected because cell therapy (target for gene transfer), a new medical practice, was born in blood transfusion services.

\section{Gene marking}

Marker genes are of great value for the accurate follow-up, both in vitro and in vivo, of transduced cells. The neomycin-resistance (neoR) gene which induces resistance to 0418 , a neomycin analog, has been used as a genetic

1 - Etablissement Français du Sang, 100 avenue de Suffren, 75015 Paris, France

2 - Etablissement Français du Sang Bourgogne Franche-Comté

Correspondence: 1 - Boulevard A. Fleming, BP 1937, 25020 Besançon cedex, France 
marker in clinical trials. More than 20 genemarking clinical trials have been performed so far, using neoR. Gene transfers and resistance to $G 418$ has been demonstrated in a very small percentage of the re-infused cells. However, use of the neoR gene is hampered by several problems, notably as neoR does not necessarily demonstrate transgene integration and/or expression.

The first gene transfer protocol which entered clinical practice involved gene marking studies. Clinical marking studies have been limited to the hematopoietic stem cell system and its progeny. Such studies have provided valuable information about stem cell biology, factors influencing gene transfer efficiency, and the mechanism of relapse in patients receiving stem cell rescue as cancer therapy. The first marking studies were undertaken in patients receiving autologous bone marrow transplantation (BMT) as part of therapy for ANIL or neuroblastoma (5). To prove that the marker gene is in leukemia cells, it is necessary to have a collateral leukemia-specific marker to show both this marker and the marker gene in the same cell. Double markers can be used to compare the short and long term repopulating ability of different sources of putative stem cells, such as peripheral blood and the marrow, or in determine the function of stem cell subpopulations, for instance CD34 4 CD38+ versus CD34+ CD 38 (4). After allogeneic BMT, gene marking studies have been used to evaluate the biological efficacy of adoptive transfer of cytotoxic T lymphocytes (CTL) directed at viral or tumor antigens (6).

Therefore, the search for transgene encoding and activity, both innocuous and easy to carry out in situ, has led to the use of genes such as the LacZ gene (7). The bacterial LacZ gene encodes $\beta$-galactosidase. In mammalian cells, the Lac $Z$ gene product is mainly found in the cytoplasm when chromogenic substrates are used for in situ detection. Cells expressing LacZ can be visualized by their cytoplasmic staining surrounding a pale lighter area. The LacZ gene was used as a reporter gene to analyze the expression of specific or ubiquitous promoters, in different cell and tissue types. All the results yet published, suggest that the use of native or modified LacZ genes does not affect cell behavior and that ( $\beta$-galactosidase is a safe marker for clinical trials).
Transplantation remains the best way to determine whether or not a cell population does contain stem cells. However, in humans, this never occurs in an experimental setting, but in a therapeutic situation which provides information a posteriori. Therefore, in vitro assays and animal models remain necessary in experimental settings.

Transfer of the LacZ gene into immunoselected CD34+ human progenitor cells using AAV-derived vectors has already been achieved (4). Up to $70 \%$ of the transduced CD 34+ cells were found to express the transgene, as assessed by X-Gal staining. It is much more difficult to infect a proportion of very immature cells, as assayed in LTC-IC assay or in the SCID-hu model, than to infect CD34+ cells or clonogenic cells.

\section{Genetransfer into hematopoietic stem cells}

Hematopoietic stem cells are interesting targets for gene transfer with potential applications for hematological, oncological or viral diseases, since the efficient transduction of stem cells should result in transduced daughter cells in all blood lineages for the lifespan of the treated individual. This goal also represents a technical challenge because of the small proportion of stem cells in hematopoietic tissues $(<0.1 \%)$, their quiescent status, the loss of expression of integrated transgene during successive divisions and differentiation, the low transduction efficiency of immature progenitors $(7,8)$.

Concerning the hematopoietic tissue, actual experimental procedures have used 3 types of target cells: unseparated bone marrow cells, mononuclear cells and CD34+ cells. Studies carried out in dogs and humans indicate that the use of long-term culture systems is efficient for transducing reconstitutive cells. This system appears, therefore, very attractive. Stromal cells, serving as a feeder layer during the transduction procedure, have been used in several studies. It has been shown that a protocol using bone marrow CD 34+ cells cocultured for 3 days with passaged stromal cells, growth factors (IL-3+IL$6+S C F)$ and viral particles being added once daily, resulted in efficient transduction of multipotent precursors. This result indicates that 
transduction of primitive human cells is possible under these conditions (4).

$A$ recent clinical protocol, involving the retrovirus-mediated transfer of $\mathrm{CDNA}$ for human glucocerebrosidase into HSC of patients with Gaucher disease, tested several parameters for ex vivo transduction (9).

Successful experiments of gene transfer into mouse HSC have stimulated the development of blood cell gene therapy models in monkeys, which would closely approximate human patients.

Fisher's team have shown, in two patients, the ability of $\gamma c$-transduced CD $34+$ cells from SCID-X 1 patients (absence of marker 1 and NK lymphocytes) to mature ex vivo into $T$ cells as well as NK cells. CD34+cells were purified using a Miltenyi medical device (97\% purity).

Alter gene transfer, $40 \%$ ( $1^{\text {st }}$ patient) and $20 \%$ $\left(2^{\text {nd }}\right.$ patient) of CD34+ cells expressed the transgene. Ten months after injection of transduced CD34+ cells, a full immune reconstitution was observed and $\gamma c$ transgene expressing $T$ and NK cells was detected in both patients (10).

Transfer of chemoresistance genes was the first attempt to transduce HSC, because of their potential to allow high-dose therapy in cancer patients. Transduction of HSC with MDR-1, MGMT, ALDH or a combination of these genes, may allow effective treatment without doselimiting myelotoxicity (14). A pilot study of MDR-1 gene transfer in patients receiving a CD34-selected peripheral blood HSC transplant for lymphoma, has been implemented. Gene transfer was documented, there was however no evidence of in vivo gene transfer, thus demonstrating the inefficiency of adult human HSC transduction using amphotropic retroviral vectors (11).

\section{«Suicide»gene and acute GvHD treatment}

Ex vivo T-cell depletion (TCD) of the graft and post-transplantation immunosuppression are the two methods presently used to prevent the deleterious effects of allogeneic reactivity after transplantation. Although very efficient in preventing GvHD, ex vivo TCD is associated with increased graft rejection and leukemia relapse as well as overall immune competence.

The ex vivo transfer of the herpes-simplex thymidine-kinase (HS-tk) gene into $\mathrm{T}$ cells before their infusion with a TCD graft, could allow for selective in vivo depletion of these $T$ cells with Ganciclovir (GCV) if subsequent GvHD developed (12). Efficient GCV-based prevention and treatment of GvHD induced by HS-tk-expressing donor $T$ cells has been demonstrated in murine models (13).

Clinical trials involving the use of HS-tkexpressing $T$ cells at time of transplantation, in conjunction with a T-cell-depleted hematopoietic graft, or subsequently for the treatment of relapse or lymphoma associated with Epstein-Barr virus infection, are currently underway (14). In vivo circulation of ganciclovirsensitive genemodified cells as well as the occurrence of ganciclovir-sensitive acute and chronic GvHD, have been documented.

The need for safe and standardized preparation conditions for gene-modified cells is crucial. In our institution, we use a closed culture system for ex vivo retrovirus-mediated gene transfer and transduced cell selection (16). Five steps are performed in the closed system: PBMC isolation and activation, PBMC retrovirus-mediated transduction, cell washing and resting period, selection of transduced cells, dead cell removal and cryopreservation of gene-modified cells (GMC) until clinical use. This closed system allows safe and reproducible ex vivo preparation of genemodified primary $\mathrm{T}$ lymphocytes for clinical use.

We have conducted a study to evaluate the feasibility of such an approach. Escalating doses of donor HS-tk-expressing CD3+ GMC were infused with a T-cell-depleted BMT. Twelve patients with hematological malignancies received $2 \times 10^{5}(n=5), 6 \times 10^{5}(n=5)$ or $20 \times 10^{5}(n=2)$ donor CD3+ GMC/kg with a BMT from an HLA-identical sibling. No acute toxicity was associated with the administration of the GMC. An early increase of circulating GMC followed by a progressive decrease and long-lasting circulation of GMC was documented. Ganciclovir treatment resulted in a significant rapid decrease of circulating GMC. Three patients developed acute grade $\geq$ II GvHD, while one patient developed chronic GvHD. Treatment with GCV alone was associated with a CR in 2 patients with acute GvHD while addition of glucocorticoids was necessary to achieve a CR in the last case. Long- 
lasting CR was achieved with GCV treatment in the patient with chronic GvHD. Unfortunately, Epstein-Barr virus lymphoproliferative disease occurred in three patients (17).

$O$ verall, the administration of low numbers of HS-tk-expressing T cells early after BMT is associated with no acute toxicity, persistent circulation of the GMC and GCV-sensitive GVHD. Such findings open the way to the infusion of higher numbers of gene-modified donor $T$ cells to enhance post-BMT immune competence preserving GCV-sensitive alloreactivity.

A clinical protocol was developed by Bordignon's team, based on the use of donor lymphocytes transduced by a retroviral vector, for transfer and expression of two genes. The HSVtk gene confers in vivo GCV sensitivity to the transduced $T$ cells, for in vivo specific elimination of cells potentially responsible for GVHD. A modified form of the low-affinity Nerve Growth Factor gene receptor is used for the in vitro selection of transduced cells and in vivo followup of the infused donor lymphocytes. In a phase I-II study, eight patients received escalating doses of donor PBMC transduced by the retroviral vector. Long-term in vivo survival of transduced cells has been demonstrated in this study, three patients developed GVHD requiring GCV treatment followed by a near resolution of all clinical and biochemical signs of acute GvHD. A potential limitation of such clinical approaches could be the development of a specific immune response against vector-encoded proteins, possibly allowing selective elimination of the transduced cells by the host immune system.

In addition to inducing anti-tumor activity, the infusion of genetically-engineered donor lymphocytes may play a role in restoring immunity against opportunistic infections early after allogeneic BMT.

\section{Genetically modified cells and immunotherapy}

\section{Donor Lymphocyte infusion}

It has been established that the curative potential of allo-BMT resides in the additional effects of high-dose chemoradiotherapy combined with the presence of allogeneic $T$ lymphocytes responsible for the graft-versus- leukemia ( $G \mathrm{vL}$ ) effect. It has been shown that delayed infusion of donor lymphocytes, alter T-cell-depleted allogeneic BMT, once graft tolerance is established, was effective in treating leukemia relapses, especially in CML and EBVinduced lymphoproliferative disorders. On the other hand, the infusion of donor lymphocytes has been shown to increase the incidence of $\operatorname{GvHD}(14,19)$. The suicide gene strategy could be applied in this clinical context by transducing all infused donor lymphocytes. The designed retroviral vectors carried a gene encoding a modified (non-functional) cell surface marker not expressed $\mathbf{0 1 1}$ human lymphocytes. Positive immunoselection of the transduced cells by the use of cell surface markers resulted in virtually $100 \%$ gene-modified lymphocytes.

\section{Immunotherapy of cancer}

Tumor cell engineering with a cytokine gene is a promising way to ensure that this cytokine will be durably present at the tumor site. The immunogenicity that tumor cells can acquire upon cytokine gene transduction may stem from recruitment by released cytokines of particular repertoires of inflammatory cells.

Vaccination with cytokine-transduced, freshly isolated cells, which should retain the tumorantigen repertoire, could be a way of generating tumorspecific $T$ lymphocytes, with which the antigens expressed by tumors other than melanoma (i.e. MAGE family genes) could be identified. Cytokineengineered tumor cells should be used as vaccines in minimal disease settings (2).

\section{Mesenchymal stem cells to deliver exogenous gene products}

Human bone marrow contains other than hematopoietic stem cells, currently referred to as mesenchymal stem cells (MSC), because of the ability in differentiate into cells that can be defined as marrow stromal cells (20). Based on this functional ability (transplantability), MSC are an attractive vehicle for gene therapy, in that they are expected not to be lost as rapidly as hematopoietic progenitors through differentiation. Examples of diseases in which stromal cell-mediated gene therapy might be appropriate include factor VIII and IX deficiencies and the various lysosomal storage 
diseases. Stromal cells might also be transduced with the cDNA of various cytokine hematopoietic growth factors (14).

\section{Gene therapy for AIDS}

Since HIV-1 predominantly infects cells of the hematopoietic system, multipotent stem cells or more mature CD4+ cells constitute potential targets for the introduction of a foreign antiviral gene that will inhibit HIV-1 replication and/ or spread (21). Re-infusion of the geneticallymodified cells into HIV-infected patients should theoretically lead to host re-population with HIV-1-resistant CD4+ cells that might be able to control in vivo virus propagation.

TAT and REV proteins, key regulatory proteins, constitute attractive targets for inhibition by gene therapy strategies. The limitations associated with the use of trans-dominant inhibitors derived from HIV-1 proteins, demonstrate the need for the development and evaluation of alternative gene therapy strategies, such as anti-sens and ribozyme RNA, or immunotherapy using adoptive transfer of natural or engineered anti-HIV-1 CTLS.

\section{Conclusion}

Transfusion medicine has demonstrated its expertise in the field of cell collection (HSC, lymphocytes, etc.) by apheresis, purification of specific cell populations, ex vivo purging, cell expansion and cryopreservation. This technical expertise can be expanded to ex vivo gene transfer techniques as well as new cellular therapies. Blood transfusion services have been described in several cases as an optimal site for cell processing, manipulation, selection and cryopreservation in closed systems (22).

The development of such novel approaches in a setting of good manufacturing practices is essential. In this respect, partnership with private industries needs to be considered. The concept of quality assurance must govern all the steps that individually or collectively influence the final quality of an ex vivo gene therapy product. Many technical advances observed in this research field are associated with our evergrowing knowledge of cell biology (i.e. human hematopoietic stem cells).
The therapeutic potential of ex vivo gene therapy is considerable. Transfusion medicine can play a significant role in supporting and promoting such innovative approaches.

\section{Novas fronteiras em terapia transfusional: terapia genética}

Patrick Hervé, Pierre Tiberghien

\section{Resumo}

No relato são apresentados aspectos relevantes dos avanços obtidos para a medicina transfusional através da terapia genética.

Rev.bras.hematol.hemoter.,2000(22)(3):362-367

Palavras-chave: Medicina transfusional, terapia genética

\section{References}

1. Hervé $P$, Tiberghien $P$. The emergen ce of cell therapy in France. Public health, regulations and other controversial issues. Hematol Cell Ther. 1997; 39: 189-192.

2. Hillyer $C D$, Klein HG. Immunotherapy and genetransfer in the treatment of the oncology patient: role of transfusion medicine. Transfus. Med. Rev 1996; 10: 1-14.

3. Blaese RM, Culver KW, Miller AD et al. T Iymphocyte-directed gene therapy for ADASC1TD: initial trial results after 4 years. Science 1995; 270: 475-480.

4. Péault B, Charbord P. Gene transfer into human haematopoietic stem cells. Transfus. Sci. 1997; 18: 291-311.

4. Bordignon $\mathrm{C}$, Notarangelo LD, Nobili $\mathrm{N}$ et al. Gene therapy in peripheral blood lymphocytes and bone marrow for ADAimmunodeficient patients. Science 1995; 270: 470-475.

5. Brenner M. Gene Marking - Review. Hum. Gene Ther. 1996; 7: 1927-1936.

6. Walter EA, Greenberg PD, Gilbert MJ et al. Reconstitution of cellular immunity against cytomegalovirus in recipients of allogeneic bone marrow by transfer of 1celi clones from the donor. N. Engl. J. Med. 1995; 333: 1038-1044.

7. Bagnis C, Gravis G, Imbert AM ey al. Retroviral transfer of the nIsLacZ gene into 
human CD34+cell populations and in to TF1 cells: future prospects in gene therapy. Hum. Gene Ther. 1994; 5: 1325-1333.

7. Goodman S, Xiao X, Donahue RE et al. Recombinant adeno-associated virusmediated gene transfer into hematopoietic progen itor cells. Blood 1994; 84: 1492-1500.

8. Richter J. Genetran sfer to hematopoietic cellsthe clinical experience. Eur. J. Haematol. 1997; 59: 67-75.

9. Dunbar C, Kohn D. Retroviral mediated transfer of the cDNA for human glu cocerebrosidase into haematopoi etic stem cells of patients with Gaucher disease. A phase 1 study. Hum. GeneTher. 1996; 7: 231-253.

10. Cavazzana-Calvo M, Hacein-Bey S, de SaintBasile $O$ et al. Genetherapy of human severe combined immunodeficiency (SCID)-XJ disease. Science 2000, in press.

11. Devereux S, Corney C, Macdonald C et al. Feasi bility of multidrug resistance (MDR- 1) gene transfer in patients undergoing highdose therapy and peripheral blood stem cell transplantation for lymphoma. Gene Ther. 1998; 5: 403-408.

12. Tiberghien P, Reynolds CW, Keller J et al. Ganciclovir treatment of herpes simplex thymidine kinase-transduced primary $T$ lymphocytes: an approach for specific in vivo donor T-cell depletion after bone marrow transplantation. Blood 1994; 84: 1333-1341.

13. Contassot E, Ferrand C, Angonin R et al. Ganciclovir-sen sitive acute graft-versus-host disease in mice receiving herpes simplex virus-thymidine kinase-expressing donor $\mathrm{T}$ celis in a bone marrow transplantation setting. Transplantation 2000; 69: 503-508.

14. Koç ON. Allay JA, Lee $K$ et al. Transfer of drug resistance genes into hematopoietic progenitors in improve chemotherapy tolerance. Semin. Oncol. 1996; 23: 46-65.

14. Bordignon C, Carlo-Stella C, Colombo MP et al. Ceil therapy: achievementsand perspectives. Haematologica 1999; 84: 1110-1149.

16. Mulligan RC. The basic science of gene therapy. Science 1993; 260: 926-932.

16. Robinet $E$, Certoux JM, Ferrand $C$ et al. A closed culturesystem for the ex vivo transduction and expansion of human T lymphocytes. J. Hematother. 1998; 7: 205-215.

17. Tiberghien $P$, Ferrand $C$, Lioure $B$ et al. Administration of herpes-simplex thymidin ekinase-expressing donor $\mathrm{T}$ cells with a T-cell-depleted allogeneic marrow graft. Paper submitted.

18. Bonini C, Ferrari G, Verzelleti S et al. HSVTK gene transfer into donor limphocytes for control of allogeneic graft-versus-leukemia.

Science 1997; 276: 1719-1724.

19. Kolb EU, Schattenberg A, Goldman et al. Graftversus-leukemia effect of donor lymphocyte transfusions in marrow grafted patients. European (group for Blood and Marrow Tansplantation Working Party Chronic Leukemia. Blood 1995; 86: 2041-2050.

20. Prockop DJ. Marrow stromal cells as stem cells for non hematopietic tissues. Science 1997; 276: 71-74.

21. Sorg I, Methali M. Gene therapy for AIDS. Transfus. Sci. 1997; 18: 277-289.

22. Tiberghien $P$, Hervé $P$. Cell and gene therapy: a future for blood banks. Theme editorial. Transf Sci. 1997; 18: 23 1-233.

Recebido: 08/06/2000

Aceito: $11 / 09 / 2000$ 